\title{
Challenges of developing communicative interaction in individuals with congenital profound intellectual and multiple disabilities
}

\section{Stefanie Blain-Moraes \& Tom Chau}

To cite this article: Stefanie Blain-Moraes \& Tom Chau (2012) Challenges of developing communicative interaction in individuals with congenital profound intellectual and multiple disabilities, Journal of Intellectual and Developmental Disability, 37:4, 348-359, DOI: 10.3109/13668250.2012.721879

To link to this article: https://doi.org/10.3109/13668250.2012.721879

Published online: 25 Sep 2012.

Submit your article to this journal $₫$

Џll Article views: 472

Q View related articles ¿

Citing articles: 18 View citing articles 


\title{
Challenges of developing communicative interaction in individuals with congenital profound intellectual and multiple disabilities
}

\author{
STEFANIE BLAIN-MORAES ${ }^{1} \&$ TOM CHAU $^{2}$ \\ ${ }^{1}$ Physical Medicine $\mathbb{E}$ Rehabilitation, University of Michigan, Ann Arbor, USA, and ${ }^{2}$ Bloorview Research Institute, \\ Holland Bloorview Kids Rehabilitation Hospital, Toronto, Canada
}

\begin{abstract}
Background Physiological responses have been used in individuals with acquired disability to enable communicative interaction without motor movement. This study explored four autonomic nervous system (ANS) signals-electrodermal activity, skin temperature, cardiac patterns and respiratory patterns - to enable interaction with individuals born with profound intellectual and multiple disabilities (PIMD).

Method A series of case studies were conducted to teach a 15-year-old individual with PIMD to voluntarily control his ANS signals for the purposes of communicative interaction. Training was carried out according to an alternating treatment single-subject research design.

Results Training was unsuccessful; however, ANS signal patterns revealed issues unique to people with PIMD: profound intrasubject variability, discrepancy between physiological responses and caregiver perspectives, and the participant's lack of contingency awareness.

Conclusions These three priority areas unique to people with congenital PIMD must be addressed before ANS signals can be used to enable communicative interaction with this population.
\end{abstract}

Keywords: access pathways, autonomic nervous system, congenital disability, profound intellectual and multiple disabilities, communicative interaction

\section{Introduction}

Advances in medical technology over the past several decades in Western industrialised countries have made it possible for individuals to survive conditions that had previously been considered catastrophic or fatal (Carnevale, Rehm, Kirk, \& McKeever, 2008). The surviving individuals are often entirely dependent on technologies and human caregivers to provide lifelong complex continuing care. Among these individuals are children who are born with severe and profound disabilities, who constitute a subpopulation with unprecedented needs and challenges: “... until 20 years ago, children this medically complex didn't exist. They didn't survive. High-tech medicine has created a new strain of human beings who require superhuman care" (Brown, 2009, p. 96).
These children are among those who are considered to have profound and multiple learning disabilities (PMLD; Bellamy, Croot, Bush, Berry, \& Smith, 2010), or profound and multiple disabilities. Herein, we adopt the term "profound intellectual and multiple disabilities" (PIMD) to describe this population, which is characterised by both (a) profound intellectual disability, and (b) profound motor disability (Nakken \& Vlaskamp, 2007). The degree of disability experienced by individuals in this group is so severe that they have little or no apparent understanding of verbal language, no apparent symbolic interactions, and practically no ability for self-support (Goldbart, 1994; Hogg \& Sebba, 1986). Furthermore, they often experience sensory impairments, especially cerebral

Correspondence: Stefanie Blain-Moraes, PhD, University of Michigan Medical School, Department of Physical Medicine and Rehabilitation, C640 Med Inn Building, 1500 East Medical Center Drive, Ann Arbor, MI 48109-5841G, USA. E-mail: sblain@umich.edu 
visual impairment (Evenhuis, Theunissen, Denkers, Verschuure, \& Kemme, 2001; Janicki \& Dalton, 1998). These functional and sensory impairments manifest in unique configurations in each child; as a result, every child has an idiosyncratic communicative repertoire. Many have never developed a consistent and reliable way of interacting with their physical and social environment. Consequently, they have minimal opportunity to engage in social interaction, compromising their ability to develop a sense of personhood and to be seen by those around them as an "other" with whom interaction is possible (Kitwood, 1997; Waksler, 2006).

Rehabilitation scientists and practitioners have devoted considerable effort to the development of access pathways via which individuals with PIMD may interact with their environment. A large variety of assistive technologies have been developed to harness even a subtle motor movement and to translate it into communication and environmental control functions (Tai, Blain, \& Chau, 2008). These technologies are suitable for individuals who are able to produce a reliable motor movement and who have the cognitive ability and motivation to learn to use technology for intentional control of their environment. In some cases, while an individual may have a reliable motor movement that can be harnessed for this purpose, they have yet to develop "contingency awareness" (Watson, 1966), or cause-and-effect learning, between this movement and consequent changes in their environment. Several training programs have been developed to facilitate the development of this awareness. Noteworthy examples include research with microswitches that allow children with PIMD to access their immediate surroundings and to activate pleasure-evoking stimuli (Lancioni, O'Reilly, \& Basili, 2001a; Lancioni et al., 2005; Lancioni, O'Reilly, et al., 2006; Lancioni, Singh, et al., 2006). Lancioni et al. (2003) demonstrated that providing microswitch environmental control does indeed facilitate the development of contingency awareness in this population. However, even these new technologies and techniques cannot be used for the subpopulation of children who do not have any form of reliable motor movement. These children's ability to interact with their environment is so limited that they are often considered to exist in a vegetative state, in spite of the fact that many exhibit signs of non-reflective consciousness and cognitive ability (Bosco et al., 2009). Recent research has begun to explore the possibility of harnessing physiological signals instead of physical signals to create potential access pathways for this subset of individuals with PIMD who may not have the physical ability to intentionally interact with their environment.

The physiological signals of both the central and autonomic nervous systems (ANS) are affected by an individual's mental and emotional state. Substantial research has been conducted, especially in the area of brain-computer interfaces (BCIs), to develop technologies that enable an individual to deliberately manipulate their physiological signals for the purposes of environmental control and communication (Birbaumer, 2006; Kübler, Kotchoubey, Kaiser, Wolpaw, \& Birbaumer, 2001; Wolpaw, Birbaumer, McFarland, Pfurtscheller, \& Vaughan, 2002). However, with a few exceptions (Birbaumer et al., 2000; Kübler, Neumann, Wilhelm, Hinterberger, \& Birbaumer, 2004; Sellers \& Donchin, 2006), most of this work has been conducted with individuals without disability. Research with individuals with disability has focused on individuals with acquired disability (e.g., in the event of a brainstem stroke) or with progressive conditions (e.g., amyotrophic lateral sclerosis). In both these cases, a baseline estimate of the individual's cognitive and comprehensive abilities can be provided from his or her history, and the individuals have had prior experience in interacting with their social and physical environment. In other words, contingency awareness between intentional thought and changes in the immediate environment has already been established. Additionally, current physiological signal-based access pathways are developed with individuals who are able to definitively convey their intended mental or emotional state. While some individuals with disability can communicate idiosyncratically within a specific context, more often than not, someone who is familiar with the individual typically acts as a third-party interpreter and assigns communicative intent (Goode, 1990; Grove, Bunning, Porter, \& Olsson, 1999; Hogg, Reeves, Roberts, \& Mudford, 2001). For individuals with acquired disability, this interpretation can be informed by knowledge of the individual's preferences and personality prior to the development of their communication difficulties. However, in individuals with congenital PIMD, caregivers do not have this source of knowledge to draw upon and therefore assign communicative intent with a high degree of uncertainty.

Consequently, current physiological signal-based access technologies cannot be readily applied in cases of congenital PIMD, who therefore remain without a pathway for communicative interaction. However, physiological signals remain very promising as a basis for future communicative pathways for this population. Vos, De Cock, Petry, Van Den Noortgate, and Maes (2010) demonstrated that 
individuals with PIMD demonstrate physiological differences in respiratory, cardiovascular, and electrodermal patterns between positive and negative emotions. Such differences could potentially be harnessed in this population to provide caregivers with a basic indication of preference, and a foundation to develop more sophisticated methods of communication. Drawing upon our own efforts to outfit a child with severe cerebral palsy with a physiological signal-based access pathway, we identify in this paper three key challenges that must be addressed before the full potential of physiological access can be realised for people with congenital PIMD. We suggest potential solutions to these challenges and future work to enable individuals with congenital PIMD to intentionally interact with their environment.

\section{Methods}

\section{Setting and design}

This study was conducted in a large, urban, paediatric rehabilitation hospital that provides longterm rehabilitation and complex continuing care to inpatient and outpatient clients with a variety of congenital and acquired disabilities. The participant's family contacted the research team expressing interest in a study that explored the possibility of using physiological signals to create an access pathway for interaction for children with PIMD. A series of case studies were conducted with the participant, following the general protocol used to train individuals to control a sensorimotor rhythmbased BCI: (1) screening sessions to determine baseline physiological signal patterns, (2) screening sessions to assess which stimuli could be used to elicit a physiological response, and (3) training sessions to develop control over one physiological signal (Felton, Radwin, Wilson, \& Williams, 2009; Neuper, Scherer, Wriessnegger, \& Pfurtscheller, 2009).

\section{Description of the participant}

The participant, who we will refer to as Rudy, was a 15-year-old boy born with severe spastic quadriplegia cerebral palsy, global developmental delay, and visual impairment secondary to birth asphyxia. His medical history included infantile seizures, ongoing respiratory and swallowing difficulties, gastrooesophageal reflux, frequent bouts of bronchiolitis, and pneumonia. Rudy was dependent on trained caregivers for all activities of daily living and was fed non-orally via a G-tube. He had marked or fluctuating increased tone throughout his extremities, and reduced or fluctuating tone in his neck and trunk. Rudy's personalised equipment included bilateral wrist splints and anklefoot orthoses (AFO) and a tilt-in-space manual wheelchair. Rudy had been involved in speechlanguage pathology services for the past 9 years. Many augmentative and alternative communication strategies and devices had been trialled in the past; to date, none had been successful at facilitating environmental control or communication, largely due to issues of fatigue and involuntary movement. Rudy would occasionally smile or purse his lips in response to affective stimuli; these responses were interpreted by his family and caregivers as indications of "like" and "dislike," respectively. However, as these behaviours were not consistent or reliable, we assumed that Rudy was functioning at a preintentional level of communication (Coupe, Barber, \& Murphy, 1988). Consent was obtained, and approval to conduct the study was granted by the research ethics board of the paediatric centre involved in his care.

\section{Establishing a baseline state}

Current physiological signal-based access pathways operate based on the principle of detecting a target physiological pattern within fluctuating baseline physiological changes (Sellers \& Donchin, 2006; Wolpaw, McFarland, Neat, \& Forneris, 1991). Physiological signals fluctuate with the natural rhythms of the human body (Hot, Naveteur, Leconte, \& Sequeira, 1999) and with a pattern idiosyncratic to each individual (Lacey \& Lacey, 1958); there is significant inter- and intrasubject variability within each signal. Consequently, it is necessary to establish a measure of baseline patterns against which target physiological signal patterns can be compared as a first step towards creating a functional access pathway.

Four physiological signals were recorded from noninvasive sensors from Thought Technology ${ }^{\circledR}(\mathrm{http}: / /$ www.thoughttechnology.com/) placed on the participant's fingers and around his chest: (1) electrodermal activity (EDA), (2) skin temperature, (3) heart rate, and (4) respiration rate. For a description of these signals and their responsiveness to mental and emotional states, the interested reader is referred to Blain, Chau, and Mihailidis (2008). During each recording session, Rudy remained in a resting, relaxed state for a duration of 5 minutes. A total of eight sessions were recorded over the course of 4 days, with four sessions recorded in the morning, and four sessions recorded in the afternoon.

Recording sessions were analysed to establish a pattern of intrasubject variability of Rudy's 
physiological signals. One feature was extracted from each 1-second segment of each physiological signal, namely: (1) the average of the first derivative of EDA (Blain, Mihailidis, \& Chau, 2008), (2) the average of the first derivative of fingertip temperature (Kistler, Mariauzouls, \& von Berlepsch, 1998), (3) the respiration length line (Ben-Shakhar \& Dolev, 1996), and (4) the blood volume pulse (BVP) length line. These four features formed a vector representing the state of the physiological signals at each second of time. When grouped together over one recording session, these feature vectors formed clusters in four-dimensional space that represented the variability within one recording session of Rudy's ANS signal patterns. Clusters were compared between sessions to determine the level of intrasubject variability of Rudy's physiological signals.

\section{Screening sessions}

The purpose of the screening sessions was to trial various stimuli to assess which had the most potential to elicit a physiological response (Felton et al., 2009; Neuper et al., 2009). The intensity of the response evoked by each stimulus would then be used to inform the choice of reward to be used in the training sessions. All four physiological signals of interest were recorded during these sessions; however, only EDA was analysed. Whereas all other physiological signals are affected by changes in both the sympathetic and parasympathetic nervous system, EDA patterns are affected solely by the sympathetic nervous system (Malmivuo \& Plonsey, 1995); consequently, EDA patterns are a reliable indicator of arousal (Boucsein, 1992). We considered the total number of electrodermal reactions (EDRs) that Rudy generated in response to a stimulus to be representative of the intensity of his affective reaction. Ten affective stimuli were chosen by Rudy's primary caregiver (his mother)-six that were believed to evoke a strong positive response, and four that were believed to evoke a strong negative response. These stimuli are presented in Table 1.

The selected stimuli were presented to Rudy in random order while his EDA was recorded. The presentation of each stimulus lasted for the full duration of 1 minute, which was felt to be sufficient given Rudy's cognitive and perceptual impairments. Intervals of rest between the presentation of sequential stimuli varied from 1 to 5 minutes, depending on the primary caregiver's assessment of when Rudy had returned to a baseline, relaxed state. Rudy did not demonstrate contingency awareness or any physiological responses
Table 1. Positive and negative affective stimuli used during screening sessions

\begin{tabular}{|c|c|}
\hline $\begin{array}{l}\text { Positive affective } \\
\text { stimuli }\end{array}$ & $\begin{array}{l}\text { Pictures of home and significant events } \\
\text { (e.g., pet dog, bar mitzvah celebrations) } \\
\text { Making music with a drum } \\
\text { Making music with a harmonica } \\
\text { Making music with a rainmaker instrumen } \\
\text { Shaking a colourful pompom } \\
\text { Watching a robot Tyrannosaurus Rex walk } \\
\text { across the table }\end{array}$ \\
\hline $\begin{array}{l}\text { Negative affective } \\
\text { stimuli }\end{array}$ & $\begin{array}{l}\text { Oral medical equipment (e.g., suction } \\
\text { machines, mask used to deliver } \\
\text { gaseous medicine) } \\
\text { Toothbrush } \\
\text { Facecloth } \\
\text { Ankle-foot orthosis (AFO) splints }\end{array}$ \\
\hline
\end{tabular}

suggesting anticipation of the subsequent stimulus. In typical screening sessions, responses to the stimuli are classified according to self-report of affective reaction or self-report of compliance to a mental task. However, in individuals with congenital PIMD, such self-report measures are not available. Thus, Rudy's response was assessed by two different proxies: (1) physiological responses in the form of EDRs, and (2) third-party reports from his primary caregiver based upon her observation of Rudy's reaction to the stimuli. The responses from both proxies were compared and used to select the most salient affective stimuli to use for the training sessions. A stimulus was considered effective if both proxies agreed on the presence of Rudy's reaction (i.e., the primary caregiver reported a reaction and EDRs were present). The strength of a response was measured by the total number of EDRs that Rudy generated during the minute the stimulus was being presented.

\section{Training sessions}

For ethical reasons, the stimuli that generated the largest positive affective responses were selected from among the six positive screening stimuli and used to train a contingent electrodermal response in Rudy. A custom software program was built which provided auditory feedback in the form of a tone whose pitch varied with Rudy's EDA. Specifically, a positive or negative change of $0.01 \mu \mathrm{S}$ was mapped respectively to an increment or decrement of one semitone in the pitch. Three training sessions were conducted based on an alternating treatment single-subject research design. Each session consisted of 5 or 10 baseline trials, and between 20 and 40 treatment trials. Each trial lasted for 5 minutes and the total number of EDRs was 
recorded at the end of each trial. During baseline trials, Rudy was provided auditory feedback of his EDA. During treatment trials, in addition to the auditory feedback, Rudy was presented with an 8-second reward upon the generation of an EDR, where the reward consisted of a variety of song clips (Treatment A) or a variety of movie clips (Treatment B). An equal number of Treatment $A$ and $B$ trials were presented and distributed in pseudorandom order within one session.

Training sessions were analysed to test the null hypothesis that the total number of EDRs did not change across trials. An increase in the total number of EDRs per trial for Treatment A and/or Treatment B was considered indicative of a successful training program. Two analyses were performed to test the null hypothesis: (1) The average number of EDRs generated per trial was compared between baseline and both Treatment A and Treatment B trials using a student's $t$-test, and (2) the slope of the best-fit line across trials, within treatment, was tested to determine whether it was significantly different from zero.

\section{Results}

Establishing a baseline state

Distributions of the features extracted from each physiological signal over eight sessions of Rudy's baseline resting state are presented in Figure 1. Summaries (mean and standard deviation) of the features over each of the eight sessions are presented in Table 2.

Both Table 2 and Figure 1 illustrate the enormous intrasubject variability in each physiological signal feature between sessions. Levene's test for equality of variance revealed a significant difference $(p<.01)$ in variance between each of the sessions for each physiological signal. These results illustrate the challenge of identifying a physiological baseline resting state for individuals with congenital
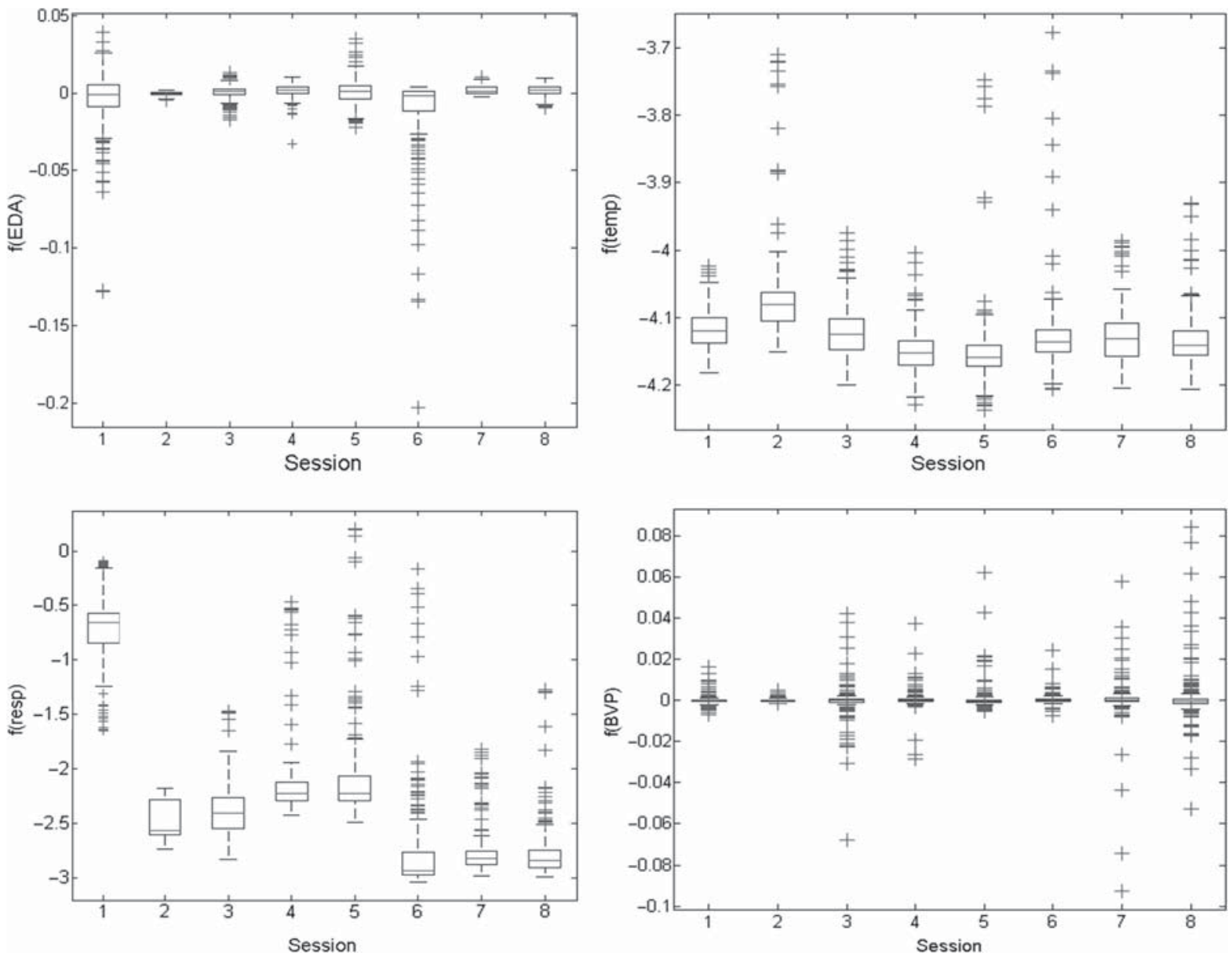

Figure 1. Distributions of features of four physiological signals collected from an individual with congenital PIMD while at rest over eight separate recording sessions. The intrasubject variability in the distribution of the features over all sessions is clearly illustrated. 
Table 2. Characteristic parameters of four physiological signals during baseline resting state

\begin{tabular}{lcccc}
\hline Session & $f(\mathrm{EDA})$ & $f($ Temperature $)$ & $f$ (Respiration) & $f(\mathrm{BVP})$ \\
\hline 1 & $-0.004 \pm 0.02$ & $-4.12 \pm 0.03$ & $-0.67 \pm 0.3$ & $-7.0 \times 10^{-5} \pm 2.0 \times 10^{-3}$ \\
2 & $-0.0006 \pm 0.001$ & $-4.07 \pm 0.06$ & $-2.5 \pm 0.2$ & $-3.0 \times 10^{-5} \pm 8.0 \times 10^{-4}$ \\
3 & $0.0006 \pm 0.004$ & $-4.12 \pm 0.04$ & $-2.4 \pm 0.2$ & $-2.0 \times 10^{-5} \pm 7.0 \times 10^{-2}$ \\
4 & $0.0016 \pm 0.004$ & $-4.15 \pm 0.06$ & $-2.2 \pm 0.3$ & $4.0 \times 10^{-4} \pm 4.0 \times 10^{-3}$ \\
5 & $0 \pm 0.008$ & $-4.13 \pm 0.06$ & $-2.1 \pm 0.4$ & $4.0 \times 10^{-4} \pm 5.0 \times 10^{-3}$ \\
6 & $-0.0095 \pm 0.02$ & $-4.13 \pm 0.04$ & $-2.8 \pm 0.2$ & $5.0 \times 10^{-4} \pm 2.0 \times 10^{-3}$ \\
7 & $0.0015 \pm 0.004$ & $-4.13 \pm 0.04$ & $-2.8 \pm 0.2$ & $8.0 \times 10^{-4} \pm 1.0 \times 10^{-2}$ \\
8 & $-0.0011 \pm 0.01$ & $-4.12 \pm 0.05$ & $-2.3 \pm 0.3$ & $3.0 \times 10^{-4} \pm 6.0 \times 10^{-3}$ \\
\hline
\end{tabular}

PIMD who have no other means of communicative interaction.

\section{Screening session}

Stimuli were considered to evoke an affective response in Rudy under the condition that both (1) an EDR, and (2) a third-party confirmation of Rudy's response were present. Rudy had the greatest affective responses to stimuli that involved both visual and auditory stimuli (e.g., the rainmaker instrument, the toy Tyrannosaurus Rex), less intensity of reaction from auditory stimuli alone (e.g., playing a harmonica), and negligible response to visual stimuli alone (e.g., pictures of family). Consequently, the two rewards that were chosen for the training sessions were movies (audiovisual), and songs (auditory).

EDA patterns for 4 of the 10 affective stimuli, and the corresponding proxy physiological and thirdparty assessment of Rudy's response are presented in Figure 2.

Figure 2 illustrates the challenge of assessing responsiveness in individuals with congenital PIMD. Under the conditions where Rudy is at rest and looking at his favourite toy, both proxies are in agreement regarding the absence or presence of his response. However, it remains unclear which source to use as the "gold standard" when they (a)

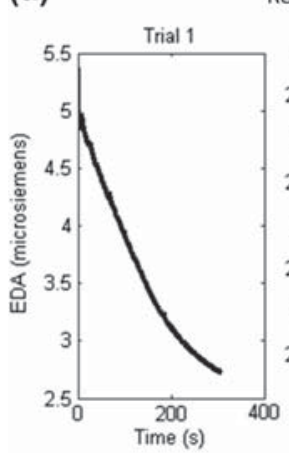

Rest
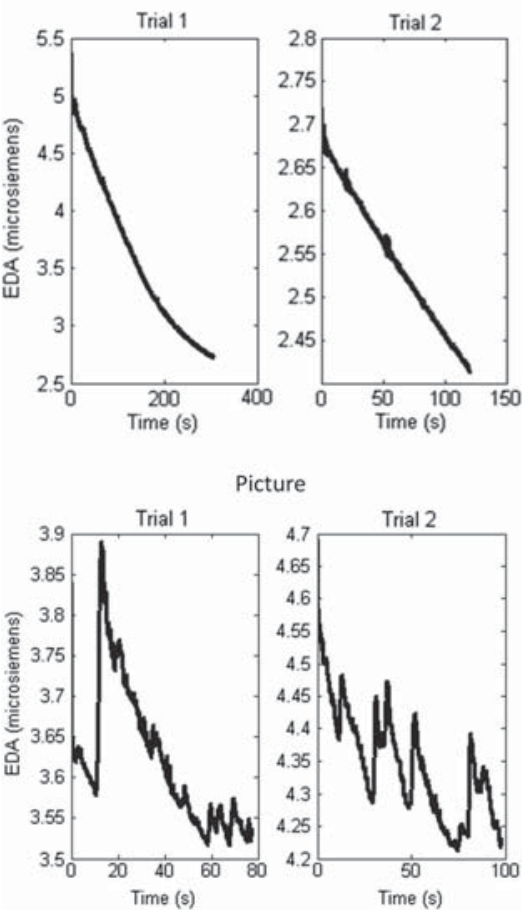

Toy
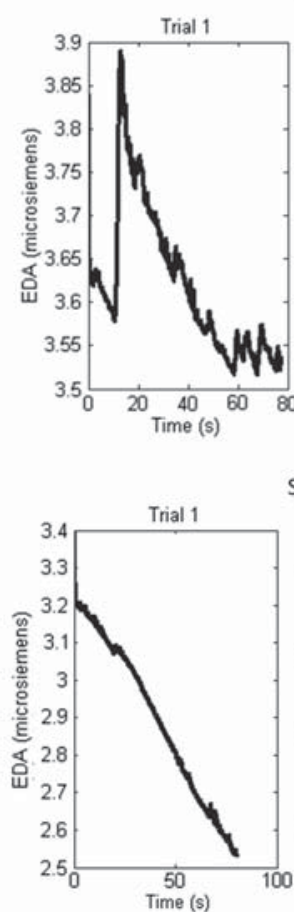

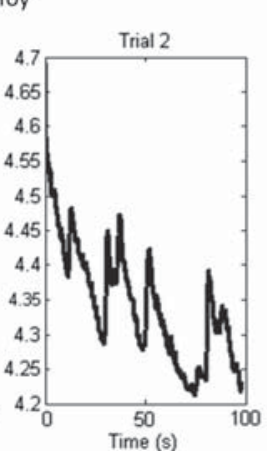

(b)

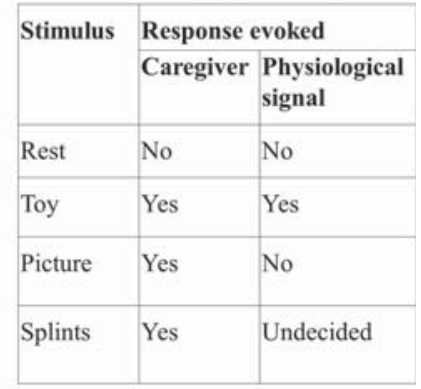

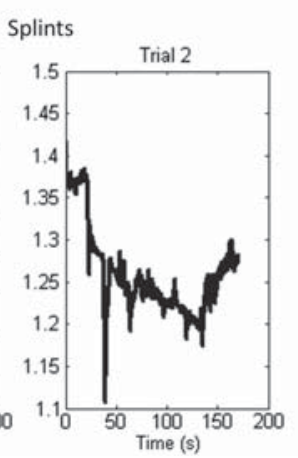

Figure 2. (a) Electrodermal activity patterns of a child with profound disabilities as he is presented with affective stimuli, as determined by his primary caregiver. This figure illustrates EDA patterns: at rest, when presented with a favourite toy, when presented with a picture of family, and when presented with medical equipment. (b) Caregiver and physiological signal assessment of whether or not each of the given stimuli evoked a response in the child. 
conflict, as in the case of the family picture and the AFO splint stimuli.

\section{Training sessions}

The total numbers of EDRs generated in each trial of each of the three training sessions are presented in Figure 3.
There were no significant differences between the average number of EDRs generated per trial during baseline and either Treatment A or B for Training Session $1(p=.20)$, Training Session $2(p=.41)$, or Training Session $3(p=.35)$. The slope of the best-fit line to both treatments was not significantly different from zero in Training Session 1 or Training Session 2, and was negative in Training Session $3(\alpha=.05)$.
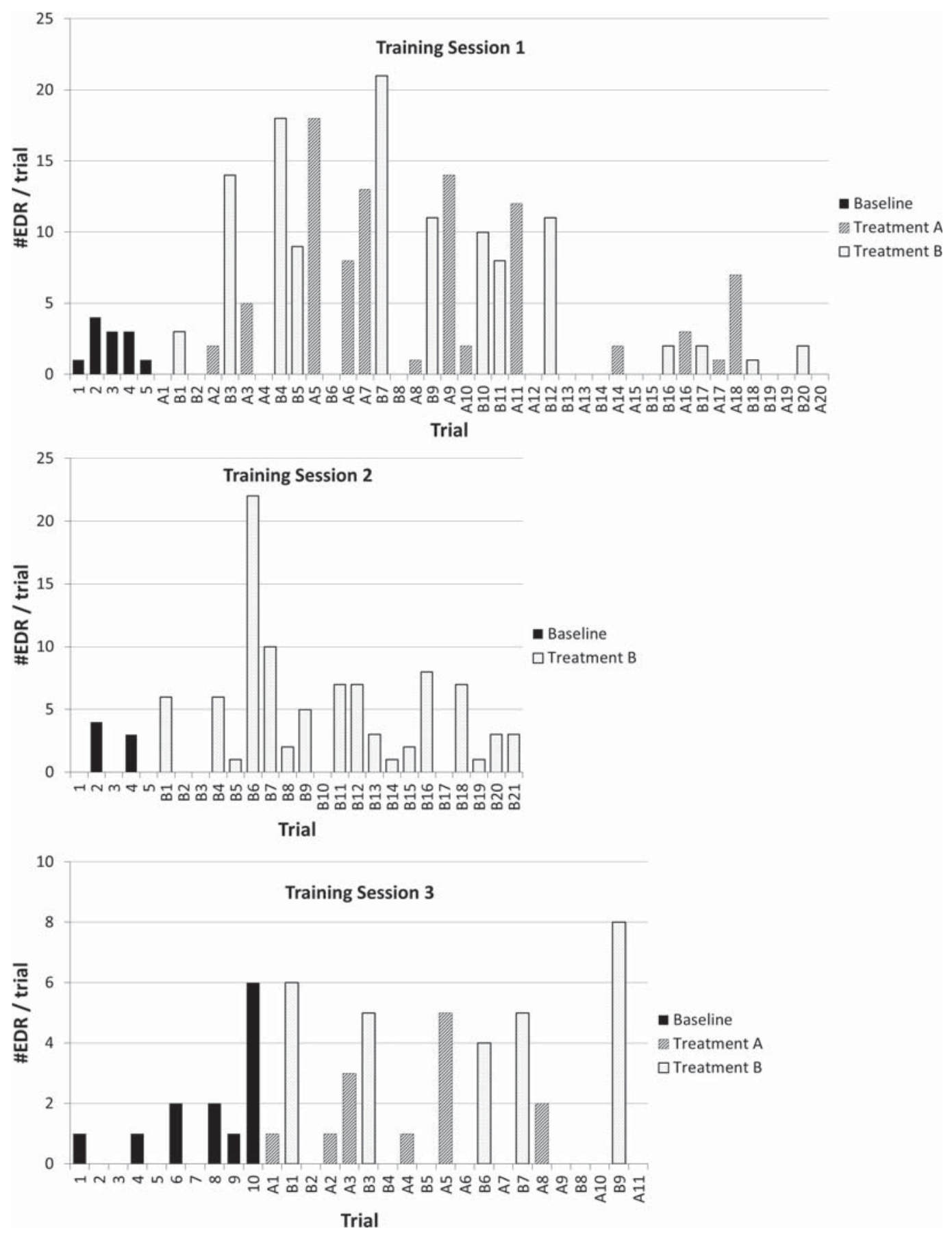

Figure 3. The total number of electrodermal reactions generated during each trial over three training sessions. No differences are observed between the total number of electrodermal reactions during baseline (solid bars) and during Treatment $\mathrm{A}$ or Treatment $B$ intervention trials in any of the three sessions. 
As a positive slope across training sessions would indicate successful learning, these results demonstrate that the training sessions were unsuccessful at teaching Rudy to voluntarily generate EDRs. While there are some trials, especially in Session 1, where the number of EDRs is almost 5 times higher than the average number of EDRs during baseline, they are succeeded by sessions where Rudy generated no EDRs. It is possible that these sessions with a high EDR frequency were simply sequential reactions to the reward stimulus, the first of which was triggered by a spontaneous EDR. These results illustrate the challenge of overcoming the lack of contingency awareness in individuals with congenital PIMD.

\section{Discussion}

Using examples drawn from efforts to outfit a child with severe spastic quadriplegia cerebral palsy with a physiological signal-based access pathway, three main challenges of developing physiological signalbased access pathways for people with congenital PIMD are illustrated: (1) the profound intrasubject variability of physiological signals, (2) the uncertainty in interpreting their responses, and (3) the lack of contingency awareness.

\section{Challenge 1: Intrasubject variability of physiological signals}

The profound intrasubject variability of physiological signals must be addressed before technologies that are able to accurately capture target physiological patterns can be developed. Current physiological signal-based access pathway technologies are typically based on algorithms that assume a baseline physiological state that remains constant in its signal characteristics. As illustrated in Table 2 and Figure 1, Rudy's baseline physiological signals differed significantly from session to session; what constitutes a significant change in state for the purposes of environmental interaction one day may be a part of his baseline state the next day.

The baseline levels of any given individual's physiological signals are affected by contextual factors such as season, time of day, and emotional state, and will change according to factors such as circadian rhythms, levels of hydration, levels of medication, levels of alertness, and eating patterns (Heywood et al., 1996; Hot et al., 1999; Mudford, Hogg, \& Roberts, 1997; Venables \& Mitchell, 1996). In recognition of this phenomenon, it is instructive to develop algorithms that continually adapt their baseline to match the changing resting physiological state of the individual. Fields such as anaesthesiology that use physiological monitoring to track the state of an individual have determined that without context-sensitive algorithms, the distinction between normal and abnormal physiological measurements is challenging and algorithms that do not address intrasubject variability often have poor accuracy (Dosani et al., 2009). For individuals with congenital PIMD who may not comprehend instructions to relax and remain in a resting state, promising approaches include developing algorithms that are recalibrated to the physiological state of the individual after events that may alter baseline state, such as eating a meal, a shift in physical positioning, or sleeping, occur.

\section{Challenge 2: Interpreting responses}

The challenge of assigning communicative meaning to the behaviours of an individual with PIMD is most often brought to light when a caregiver's interpretation of an individual's response is not empirically substantiated; this situation is clearly illustrated in Figure 2. When interpretations conflict, it is not clear which source should be used as a "gold standard" upon which to train an algorithm designed to recognise an individual's responses. Conflicting interpretations of the responses of people with congenital PIMD are commonplace; different parties involved with the child often generate diametrically opposite "versions" of the child, leading to antithetical claims about their capabilities and responsiveness (Goode, 1990).

While this challenge arises in all individuals with congenital PIMD without an established means of communication, it is especially pronounced for those at the stage of preintentional communication (Bates, 1976). For individuals functioning at a preintentional level, the development of communication hinges upon having another individual detect, attach meaning to, and respond to their reactions to a variety of internal and external stimuli (Coupe et al., 1988). As we begin to consider physiological patterns in the development of systems to detect and attach meaning to these responses, the relative importance assigned to physiological and caregiver interpretations reflects the researcher's perspective towards communicative interaction. Prioritising the physiological interpretation frames communicative competencies as an individual attribute as opposed to an attribute of the social system. In everyday interaction, the understandings of the intent and preferences of a child with profound congenital disability often arise from the mutual understanding of the details of a task at hand; in other words, 
the sharing of a routine (Goode, 1990). Within the context of a routine, the limited expressions of these individuals have the potential to be transformed into gestures with specific, context-relevant content. Similarly, shared knowledge about spatial arrangements and objects provide context through which to interpret expression and preference. The frequent misunderstanding of needs and preferences of these individuals often arises from the idiosyncratic and context-bound nature of their responses (Grove et al., 1999; Hogg et al., 2001). The conflict between the interpretation of Rudy's caregiver and that afforded by the physiological signal responses may not be a consequence of one source being "right" and the other "wrong," but the result of fundamentally different views on the nature of his communicative interactions. In future work, the challenge of interpretation could potentially be addressed by presenting Rudy with affective stimuli within his natural routine and space. The interpretation of physiological responding could then be augmented with the knowledge of these contextual factors. By adopting this approach, caregiver and physiological interpretations of responses might become complementary, rather than contradictory sources of information that might help to derive a more accurate interpretation of the mental and emotional state of individuals with congenital PIMD.

\section{Challenge 3: Lack of contingency awareness}

In this study, Rudy did not demonstrate the ability to learn to control his EDA after three training sessions. Studies have demonstrated that EDA can be trained only after participants have developed contingency awareness between their specific physiological state and the environmental feedback of that state. No evidence of conditioning has been found in the unaware participant with any measures, including self-rating psychophysiological measures and behavioural indices (Dawson, Rissling, Schell, \& Wilcox, 2007). Although those with acquired disability or progressively degenerative disability have experienced a connection between their thoughts and changes in their environment and therefore understand the possibility of this connection, it is difficult for those with congenital PIMD to develop this contingency awareness (Basil, 1992). Due to a severely limited motor repertoire, the means of communicative interaction for people with PIMD is highly compromised. Therefore, they may never have developed the awareness that they have the ability to affect the environment. This can lead to learned helplessness, a condition which has significant implications for any access pathway provided for individuals with congenital
PIMD (Basil, 1992). When a means of interacting with the environment (e.g., a mechanical switch) is provided for these individuals, responses are often variable and inconsistent. In a review of switch use in persons with disability, Lancioni, O'Reilly, and Basili (2001b) reported that eight of the 23 participants did not show differential rates of switch use across the conditions designed to determine whether or not the switch use was volitional. While this variability of responding might be attributable to the side effects of medication, medical conditions such as seizures, fatigue, and positional discomfort (Saunders et al., 2003), it is important not to rule out learned helplessness as a contributing factor.

It is difficult to determine whether Rudy's unsuccessful training sessions were the result of an innate inability to voluntarily control his EDA or a lack of contingency awareness between his thoughts and changes in his environment. To address this challenge, the authors recommend testing the two components independently: first, by establishing contingency awareness with the child, and second, by training the child to voluntarily control a physiological signal to affect the environment. While the second step is covered extensively in the BCI literature (Kübler et al., 2004; Neumann \& Kübler, 2003), the first step has received much less attention. Simple switch-based interventions, such as those introduced by Lancioni, have been established to teach children with congenital PIMD contingency awareness (Lancioni et al., 2008). In the absence of such technologies, Intensive Interaction has been used as a method of encouraging and enriching the quality of interaction between an individual with PIMD and his and her environment (Nind \& Hewett, 1994). This approach is characterised by regular, frequent interactions between an individual with profound disability and an interaction partner with no particular task or outcome focus; it is only the quality of the interaction itself that matters. Studies that have measured six categories of social behaviour (eye contact, looking at face, smiling, contingent vocalisation, joint focus, and "engaged social interaction") have demonstrated significant increases in socially interactive behaviours as a result of the Intensive Interaction program (Kellett, 2005). As an awareness of the ability to interact with their environment develops, these individuals engage in more and more activities that illustrate contingency awareness between their actions and the state of their environment.

The authors recommend that strategies such as simple switch-based interventions and Intensive Interaction be employed with individuals with congenital PIMD whose communicative interaction 
abilities are being developed. As it is crucial to develop contingency awareness before learned control of physiological signals such as EDA can occur (Dawson et al., 2007), this process is critical in the development of a successful physiological signalbased access pathway.

\section{Conclusion}

While physiological signal-based access pathways are emerging as promising ways of enabling communicative interaction, the majority of these technologies have been developed with either able-bodied individuals or on individuals with acquired or degenerative disability. A series of case studies documenting efforts to outfit a child with severe spastic quadriplegia cerebral palsy with a physiological signal-based access pathway illustrates the challenges of translating these technologies to individuals with congenital PIMD. The challenges of profound intrasubject variability of physiological signals, unambiguous interpretation of an individual's responses, and teaching contingency awareness must be addressed before these access pathways can be successfully deployed as a means of communicative and environmental interaction.

\section{Author note}

The authors would like to acknowledge the financial support from the National Science and Engineering Research Council to Stefanie Blain-Moraes.

\section{Acknowledgement}

The authors would like to thank Rudy and his family for their continued support of this work and for their contributions to this study, and Madeleine Shen and Pierre Duez for development of the biofeedback training program and their assistance with data collection.

Declaration of interest: The authors report no conflicts of interest. The authors alone are responsible for the content and writing of the paper.

This funding body has not imposed any restrictions on free access to or publication of the research data.

\section{References}

Basil, C. (1992). Social interaction and learned helplessness in severely disabled children. Augmentative and Alternative Communication, 8, 188-199. doi:10.1080/07434619212331276183
Bates, E. (1976). Language and context:The acquisition of pragmatics. New York, NY: Academic Press.

Bellamy, G., Croot, L., Bush, A., Berry, H., \& Smith, A. (2010). A study to define: Profound and multiple learning disabilities (PMLD). Fournal of Intellectual Disabilities, 14, 221-235. doi:10.1177/1744629510386290

Ben-Shakhar, G., \& Dolev, K. (1996). Psychophysiological detection through the guilty knowledge technique: Effect of mental countermeasures. Fournal of Applied Psychology, 81, 273-281. doi:10.1037/0021-9010.81.3.273

Birbaumer, N. (2006). Brain-computer-interface research: Coming of age [Editorial]. Clinical Neurophysiology, 117, 479-483. doi:10.1016/j.clinph.2005.11.002

Birbaumer, N., Kübler, A., Ghanayim, N., Hinterberger, T., Perelmouter, J., Kaiser, J., ... Flor, H. (2000). The thought translation device (TTD) for completely paralyzed patients. IEEE Transactions on Rehabilitation Engineering, 8, 190-193.

Blain, S., Chau, T., \& Mihailidis, A. (2008). Peripheral autonomic signals as access pathways for individuals with severe disabilities: A literature appraisal. The Open Rehabilitation fournal, 1(11), 27-37. doi:10.2174/1874943700801010027

Blain, S., Mihailidis, A., \& Chau, T. (2008). Assessing the potential of electrodermal activity as an alternative access pathway. Medical Engineering E゚Physics, 30, 498-505. doi:10.1016/j. medengphy.2007.05.015

Bosco, A., Lancioni, G.E., Belardinelli, M.O., Singh, N.N., O’Reilly, M.R., \& Sigafoos, J. (2009). Learning as a possible sign of non-reflective consciousness in persons with a diagnosis of vegetative state and pervasive motor impairment. Cognitive Processing, 10, 355-359. doi:10.1007/s10339-009-0334-3

Boucsein, W. (1992). Electrodermal activity. New York, NY: Plenum Press.

Brown, I. (2009). The boy in the moon: A father's search for his disabled son. Toronto: Random House Canada.

Carnevale, F.A., Rehm, R.S., Kirk, S., \& McKeever, P. (2008). What we know (and do not know) about raising children with complex continuing care needs. Fournal of Child Health Care, 12, 4-6. doi:10.1177/1367493508088552

Coupe, J., Barber, M., \& Murphy, D. (1988). Affective communication. In J. Coupe \& J. Goldbart (Eds.), Communication before speech: Normal development and impaired communication (pp. 31-47). New York, NY: Croom Helm.

Dawson, M.E., Rissling, A.J., Schell, A.M., \& Wilcox, R. (2007). Under what conditions can human affective conditioning occur without contingency awareness? Test of the evaluative conditioning paradigm. Emotion, 7, 755-766. doi:10.1037/ 1528-3542.7.4.755

Dosani, M., Lim, J., Yang, P., Brouse, C., Daniels, J., Dumont, G., \& Ansermino, J.M. (2009). Clinical evaluation of algorithms for context-sensitive physiological monitoring in children. British fournal of Anaesthesia, 102, 686-691. doi:10.1093/bja/aep045

Evenhuis, H.M., Theunissen, M., Denkers, I., Verschuure, H., \& Kemme, H. (2001). Prevalence of visual and hearing impairment in a Dutch institutionalized population with intellectual disability. Fournal of Intellectual Disability Research, 45, 457-464. doi:10.1046/j.1365-2788.2001.00350.x

Felton, E.A., Radwin, R.G., Wilson, J.A., \& Williams, J.C. (2009). Evaluation of a modified Fitts law brain-computer interface target acquisition task in able and motor disabled individuals. fournal of Neural Engineering, 6(5), 056002. doi:10.1088/17412560/6/5/056002

Goldbart, J. (1994). Opening the communication curriculum to students with PMLDs. In J. Ware (Ed.), Educating children with profound and multiple learning difficulties. (pp. 15-62). London: Fulton. 
Goode, D.A. (1990). On understanding without words: Communication between a deaf-blind child and her parents. Human Studies, 13, 1-37. doi:10.1007/BF00143039

Grove, N., Bunning, K., Porter, J., \& Olsson, C. (1999). See what I mean: Interpreting the meaning of communication by people with severe and profound intellectual disabilities. Fournal of Applied Research in Intellectual Disabilities, 12, 190-203. doi:10.1111/j.1468-3148.1999.tb00076.x

Heywood, P., Murphy, K., Corfield, D.R., Morrell, M.J., Howard, R.S., \& Guz, A. (1996). Control of breathing in man; insights from the 'locked-in' syndrome. Respiration Physiology, 106, 13-20. doi:10.1016/0034-5687(96)00060-6

Hogg, J., Reeves, D., Roberts, J., \& Mudford, O.C. (2001). Consistency, context and confidence in judgements of affective communication in adults with profound intellectual and multiple disabilities. Fournal of Intellectual Disability Research, $45,18-29$.

Hogg, J., \& Sebba, J. (1986). Profound retardation and multiple impairment (Vol. 1). London, UK: Croom-Helm.

Hot, P., Naveteur, J., Leconte, P., \& Sequeira, H. (1999). Diurnal variations of tonic electrodermal activity. International fournal of Psychophysiology, 33, 223-230. doi:10.1016/S0167-8760 (99)00060-4

Janicki, M.P., \& Dalton, A.J. (1998). Sensory impairments among older adults with intellectual disability. Fournal of Intellectual $\mathbb{E}$ Developmental Disability, 23, 3-11. doi:10.1080/136682 59800033541

Kellett, M. (2005). Catherine's legacy: Social communication development for individuals with profound learning difficulties and fragile life expectancies. British fournal of SpecialEducation,32, 116-121. doi:10.1111/j.0952-3383.2005. 00383.x

Kistler, A., Mariauzouls, C., \& von Berlepsch, K. (1998). Fingertip temperature as an indicator for sympathetic responses. International fournal of Psychophysiology, 29, 35-41. doi:10.1016/ S0167-8760(97)00087-1

Kitwood, T. (1997). Dementia reconsidered: The person comes first. Buckingham, UK: Open University Press.

Kübler, A., Kotchoubey, B., Kaiser, J., Wolpaw, J.R., \& Birbaumer, N. (2001). Brain-computer communication: Unlocking the locked in. Psychological Bulletin, 127, 358-375. doi:10.1037/ 0033-2909.127.3.358

Kübler, A., Neumann, N., Wilhelm, B., Hinterberger, T., \& Birbaumer, N. (2004). Predictability of brain-computer communication. Fournal of Psychophysiology, 18, 121-129. doi:10.1027/0269-8803.18.23.121

Lacey, J.I., \& Lacey, B.C. (1958). Verification and extension of the principle of autonomic response-stereotypy. American fournal of Psychology, 71, 50-73. doi:10.2307/1419197

Lancioni, G.E., Abels, J., Wilms, E.H., Singh, N.N., O'Reilly, M.F., \& Groeneweg, J. (2003). Microswitch responding and awareness of contingency in persons with profound multiple disabilities. Perceptual \& Motor Skills, 96, 835-838. doi:10. 2466/pms.2003.96.3.835

Lancioni, G.E., O’Reilly, M.F., \& Basili, G. (2001a). An overview of technological resources used in rehabilitation research with people with severe/profound and multiple disabilities. Disability and Rehabilitation, 23, 501-508. doi:10.1080/ 09638280010010705

Lancioni, G.E., O'Reilly, M.F., \& Basili, G. (2001b). Use of microswitches and speech output systems with people with severe/profound intellectual or multiple disabilities: A literature review. Research in Developmental Disabilities, 22, 21-40. doi:10.1016/S0891-4222(00)00064-0

Lancioni, G.E., O’Reilly, M.F., Singh, N.N., Oliva, D., Coppa, M.M., \& Montironi, G. (2005). A new microswitch to enable a boy with minimal motor behavior to control environmental stimulation with eye blinks. Behavioral Interventions, 20, 147-153. doi:10.1002/bin. 185

Lancioni, G.E., O'Reilly, M.F., Singh, N.N., Sigafoos, J., Oliva, D., Antonucci, M., ... Basili, G. (2008). Microswitchbased programs for persons with multiple disabilities: An overview of some recent developments. Perceptual \& Motor Skills, 106, 355-370. doi:10.2466/pms.106.2.355-370

Lancioni, G.E., O’Reilly, M.F., Singh, N.N., Sigafoos, J., Tota, A., Antonucci, M., \& Oliva, D. (2006). Children with multiple disabilities and minimal motor behavior using chin movements to operate microswitches to obtain environmental stimulation. Research in Developmental Disabilities, 27, 290-298. doi:10.1016/j.ridd.2005.02.003

Lancioni, G.E., Singh, N.N., O'Reilly, M.F., Sigafoos, J., Oliva, D., Costantini, A., ... Putzolu, A. (2006). An optic micro-switch for an eyelid response to foster environmental control in children with minimal motor behaviour. Pediatric Rehabilitation, 9, 53-56. doi:10.1080/13638490500074550

Malmivuo, J., \& Plonsey, R. (1995). The electrodermal response. In J. Malmivuo \& R. Plonsey (Eds.), Bioelectromagnetism: Principles and applications of bioelectric and biomagnetic fields (pp. 428-436). New York, NY: Oxford University Press. doi:10.1093/acprof:oso/9780195058239.003.0027

Mudford, O.C., Hogg, J., \& Roberts, J. (1997). Interobserver agreement and disagreement in continuous recording exemplified by measurement of behavior state. American fournal on Mental Retardation, 102, 54-66.

Nakken, H., \& Vlaskamp, C. (2007). A need for a taxonomy for profound intellectual and multiple disabilities. fournal of Policy and Practice in Intellectual Disabilities, 4, 83-87. doi:10.1111/j.1741-1130.2007.00104.x

Neumann, N., \& Kübler, A. (2003). Training locked-in patients: A challenge for the use of brain-computer interfaces. IEEE Transactions on Neural Systems and Rehabilitation Engineering, 11, 169-172.

Neuper, C., Scherer, R., Wriessnegger, S., \& Pfurtscheller, G. (2009). Motor imagery and action observation: Modulation of sensorimotor brain rhythms during mental control of a brain-computer interface. Clinical Neurophysiology, 120, 239-247. doi:10.1016/j.clinph.2008.11.015

Nind, M., \& Hewett, D. (1994). Access to communication: Developing the basics of communication in people with severe learning difficulties through intensive interaction. London, UK: Fulton.

Saunders, M.D., Timler, G.R., Cullinan, T.B., Pilkey, S., Questad, K.A., \& Saunders, R.R. (2003). Evidence of contingency awareness in people with profound multiple impairments: Response duration versus response rate indicators. Research in Developmental Disabilities, 24, 231-245. doi:10.1016/S08914222(03)00040-4

Sellers, E.W., \& Donchin, E. (2006). A P300-based braincomputer interface: Initial tests by ALS patients. Clinical Neurophysiology, 117, 538-548. doi:10.1016/j.clinph.2005. 06.027

Tai, K., Blain, S., \& Chau, T. (2008). A review of emerging access technologies for individuals with severe motor impairments. Assistive Technology, 20, 204-219. doi:10.1080/10400435.200 8.10131947

Venables, P.H., \& Mitchell, D.A. (1996). The effects of age, sex and time of testing on skin conductance activity. Biological Psychology, 43, 87-101. doi:10.1016/0301-0511(96)05183-6

Vos, P., De Cock, P., Petry, K., Van Den Noortgate, W., \& Maes, B. (2010). Do you know what I feel? A first step towards a physiological measure of the subjective well-being of persons with profound intellectual and multiple disabilities. Fournal of 
Applied Research in Intellectual Disabilities, 23, 366-378. doi:10.1111/j.1468-3148.2010.00553.x

Waksler, F.C. (2006). Analogues of ourselves: Who counts as an other? Human Studies, 28, 417-429. doi:10.1007/s10746-0059007-0

Watson, J.S. (1966). The development and generalization of "contingency awareness" in early infancy: Some hypotheses. Merrill-Palmer Quarterly, 12, 123-135.
Wolpaw, J.R., Birbaumer, N., McFarland, D.J., Pfurtscheller, G., \& Vaughan, T.M. (2002). Brain-computer interfaces for communication and control. Clinical Neurophysiology, 113, 767-791. doi:10.1016/S1388-2457(02)00057-3

Wolpaw, J.R., McFarland, D.J., Neat, G.W., \& Forneris, C.A. (1991). An EEG-based brain-computer interface for cursor control. Electroencephalography and Clinical Neurophysiology, 78, 252-259. doi:10.1016/0013-4694(91)90040-B 